\title{
Automatic feedrate adjustment for pocket machining
}

\author{
Seok-Hyung Bae, Keyhoon Ko, Bo H. Kim, Byoung K. Choi* \\ Department of Industrial Engineering, KAIST, 373-1 Guseong-dong, Yuseong-gu, Daejeon, 305-701, Republic of Korea
}

Received 9 February 2001; revised 15 October 2001; accepted 16 October 2001

\begin{abstract}
As high-speed machining and unmanned machining become common, the demand for cutting-load regularization increases, so NC machining can be more efficient. To be presented is a simple cutting-load regularization method for pocket machining. As the conventional off-line approaches where cutting-load is predicted and cutting parameters are adjusted before actual cutting, the proposed method requires a cutting force model, which is quite simplified with the function of two independent variables. One is the geometric measure so called $2 \mathrm{D}$ chip-load (cutter-engagement angle or effective cutting depth), and the other is the feedrate. Based on the 2D chip-load analysis for the concave line-line segment of the NC tool path, the adjusted feedrate is calculated by using the simplified-cutting force model (SCFM) obtained by the cutting experiment with a tool dynamometer. The concept of the automatic feedrate adjustment (AFA) method to be proposed is very simple, and the implementation requires little effort. Furthermore, the proposed method does not need much calculation time because there are no complex calculations or cutting simulation.
\end{abstract}

(C) 2002 Elsevier Science Ltd. All rights reserved.

Keywords: Cutting-load regularization; Cutting force model; 2D chip-load; Automatic feedrate adjustment (AFA); Pocket machining

\section{Introduction}

High-speed machining and unmanned machining have been widely used in manufacturing industries to improve the efficiency of NC machining. However, unwanted and/ or unexpected cutting overload, which usually occurs in actual NC machining, makes it difficult to obtain the desired level of machining efficiency. Thus, for NC machining to become more efficient, it is required to adopt the cuttingload regularization procedure to track cutting-load while machining and to adjust cutting parameters (i.e. feedrate and cutting speed, etc.) so that the cutting-load does not exceed a specified limit.

Much research has been done on cutting-load regularization. The research is classified into two categories: (1) the on-line approach and (2) the off-line approach. In on-line approaches, various types of physical sensors are used to estimate cutting-load and to adjust cutting parameters 'while' machining [1,2]. This type of method has serious problems of robustness, which are caused by the time lag of input signals. On the other hand, off-line approaches predict cutting-load by using cutting force models and adjust cutting parameters 'before' actual machining. Cutting force models that have been proposed so far, are classified

\footnotetext{
* Corresponding author. Tel.: +82-42-869-3115; fax: +82-42-869-3110.
}

E-mail address: bkchoi@vmslab.kaist.ac.kr (B.K. Choi). as follows: (1) the infinitesimal flute model and (2) the mean cutting force model. The infinitesimal flute model divides each flute of a cutter into a large number of small flutes and calculates the cutting force to the cutter by summing up the cutting force to each divided flute [3,4]. Many researchers are still struggling with the infinitesimal flute model because (1) the model is very sensitive to its parameters, (2) it is not easy to obtain the exact geometry of infinitesimal flutes from the cutters used in machining industries, and (3) the dynamics of infinitesimal flutes are not clearly understood. The mean cutting force model is based on the material removal volume (MRV) [5-7]. The MRV is calculated with the cutting simulation in which the in-process shape of the part geometry is successively updated. In spite of the drawback - the difficulty to catch instantaneous cutting force change, the mean cutting force model is adopted by some commercial CAM systems because of its simple calculation [5].

In pocket machining, if the tool path and the outer boundary of a machined region on a cutting plane are given, the inprocess shape of the part geometry can be easily predicted using the concept of the 2D cutting simulation-the inprocess shape at the current cutter-location path (CL-path) is the offset curve of the previous CL-path [8]. In addition, all CL-paths have the same nominal cutting depth (or path interval) except the inner-most path.

To be presented in the paper is a simple cutting-load 


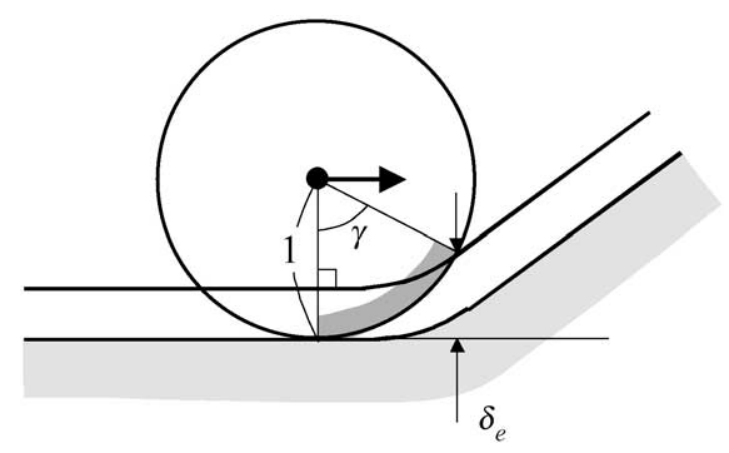

Fig. 1. Definition of 2D chip-loads.

regularization method for pocket machining, which is a kind of off-line approach. We assume that the cutting force is a function of two major independent variables. One is the geometric measure so called 2D chip-load, and the other is the physical measure, the feedrate-one of the most important cutting parameters affecting the cutting-load while machining. From the geometric analysis of a 2D chip-load, the change of chip-load along the tool path can be clarified. On the other hand, the simplified-cutting force model (SCFM), which is represented in the form of a nonparametric Bezier surface, is constructed through cutting experiments. Then, it is possible to adjust the feedrate of each NC block to maintain the cutting force below a specified limit using the SCFM based on the results of the 2D chip-load analysis.

The organization of the paper is as follows: the 2D chipload is defined and geometrically analyzed in Section 2. Presented in Section 3 is the construction procedure of the SCFM, which is used in the automatic feedrate adjustment (AFA) procedure explained in Section 4. To show the applicability of the proposed AFA method to pocket machining, an illustrative example is given in Section 5 followed by discussion and conclusion in Section 6 .

\section{2D chip-load analysis}

As noted earlier, the 2D chip-load is the geometric measure of cutting-load for 2D cutting such as pocket machining. In this study, two types of chip-loads are considered as follows: (1) the cutter-engagement angle $\gamma$, and (2) the effective cutting depth $\delta_{\mathrm{e}}$ (see Fig. 1). The cutterengagement angle relates to the contact area between a cutter and cutting material, whereas the effective cutting depth is an instantaneous MRV. For notational simplicity, the effective cutting depth is normalized with respect to the cutter radius. The cutter-engagement angle and (normalized) effective cutting depth satisfy the following relationship.

$\delta_{\mathrm{e}}=1-\cos \gamma$

The tool path (or CL-path) composed of line NC blocks (or CL-lines) can be divided into overlapped line-line segments. And each line-line segment can be classified
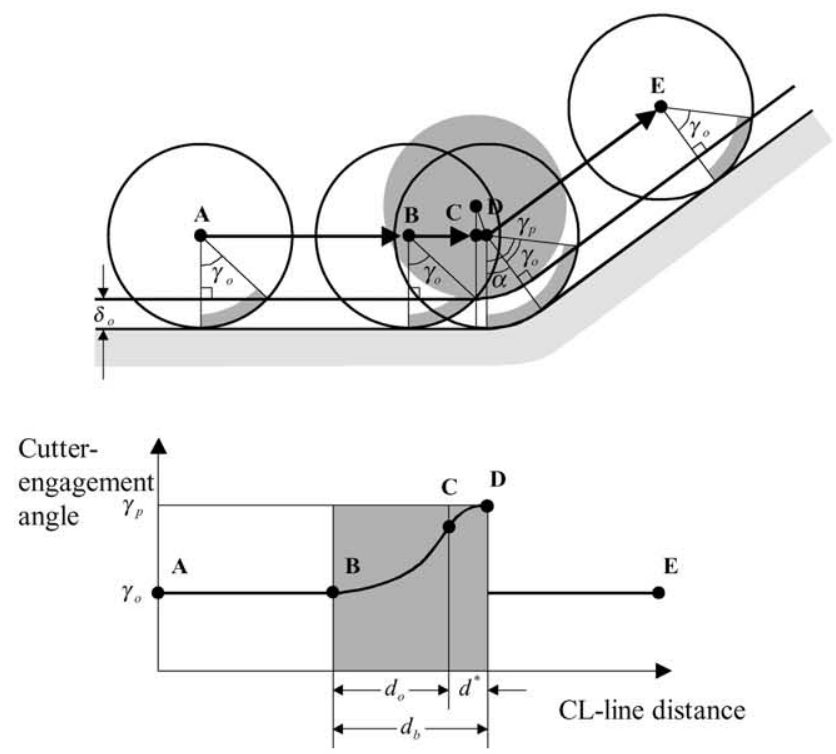

(a)
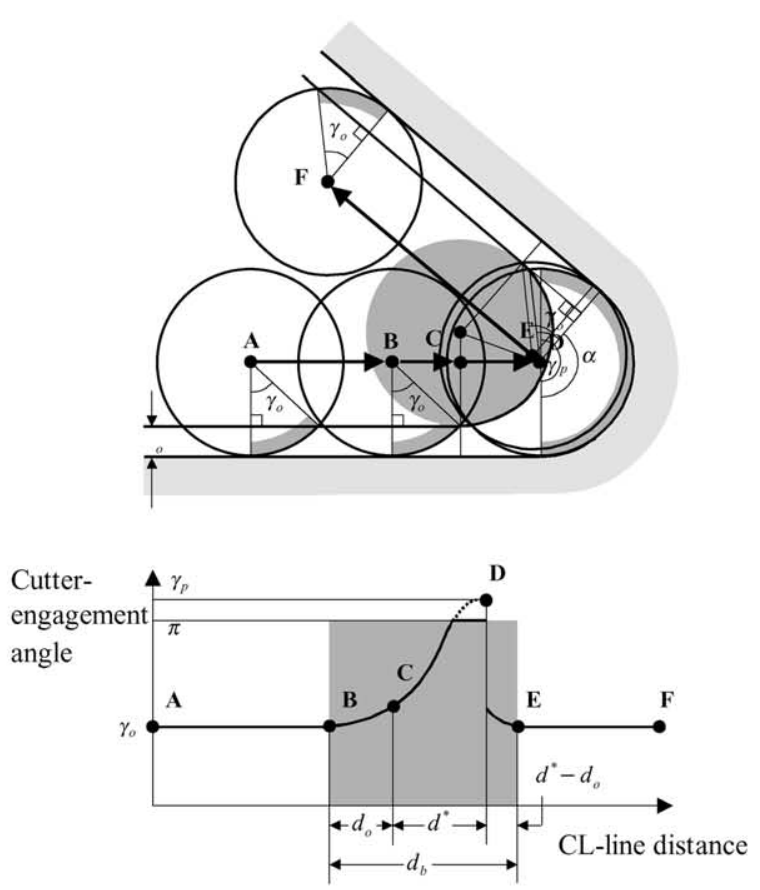

(b)

Fig. 2. Change of 2D chip-load in concave line-line segment.

into a concave segment or convex segment. Cutting overload occurs only in machining the concave segment because the two defined chip-load measures increase gradually as shown in Fig. 2 .

As shown in Fig. 2, the connection point of two CL-lines of the concave line-line segment is a concave point, and the angle between the two CL-lines is a concave angle $\alpha$. According to the degrees of the concave angle, each lineline segment is classified again into the following two types: (1) the independent type - the cutter moving along the first 
Table 1

Summary of 2D chip-load analysis for concave line-line segment

\begin{tabular}{lll}
\hline & Independent type & Dependent type \\
\hline Classification criteria & $\alpha \geq 2 \tan ^{-1}\left[\sqrt{\delta_{0}\left(2-\delta_{0}\right)} / \delta_{0}\right]$ & $\alpha<2 \tan ^{-1}\left[\sqrt{\delta_{0}\left(2-\delta_{0}\right)} / \delta_{0}\right]$ \\
Peak cutter-engagement angle $\left(\gamma_{\mathrm{p}}\right)$ & $\min \left\{\alpha+\gamma_{0}, \pi\right\}$ where $\gamma_{0}=\cos ^{-1}\left(1-\delta_{0}\right)$ & $\min \left\{\pi+(\alpha / 2)-\cos { }^{-1}\left[\delta_{0} / 2 \cos (\alpha / 2)\right], \pi\right\}$ \\
Braking distance $\left(d_{\mathrm{b}}\right)$ & $\sqrt{\delta_{0}\left(2-\delta_{0}\right)}+\delta_{0} \tan (\alpha / 2)$ & $2 \delta_{0} \tan (\alpha / 2)$ \\
\hline
\end{tabular}

CL-line does not invade the area to be machined by the cutter along the second CL-line, and (2) the dependent type - the cutter moving along the first CL-line invades the second machined area. In the independent type (see Fig. 2a), the cutter-engagement angle increases gradually until the cutter reaches the concave point, in the section called the transient range, and maintains a constant value in the other range. The length of the transient range is called the braking distance $d_{\mathrm{b}}$ because the feedrate corresponding to the transient range is required to be reduced so that the peak cutting force does not exceed the specified limit. On the other hand, the dependent type has the additional braking distance $\overline{\mathrm{DE}}$ because the transient range remains after the cutter reaches the concave point (see Fig. 2b). Summarized in Table 1 are the results of the 2D chip-load analysis for the concave line-line segment [9]. Where the symbols $\delta_{0}$ and $\gamma_{0}$ indicate the nominal values of the effective cutting depth and cutterengagement angle, respectively.

\section{Simplified-cutting force model}

In the study, we propose the SCFM, which is a singlevalued function having two independent variables. One is the geometric measure, and the other is the physical measure. As the geometric measure, the 2D chip-load (cutter-engagement angle or effective cutting depth) is used, and as the physical measure (i.e. cutting parameters), the feedrate is chosen. The revolution-per-minute (RPM), another theoretical control variable of cutting-load, is not selected as the physical variable because the change of the RPM requires the spindle gear change while machining, which may cause machined-surface quality deterioration.

Shown in Table 2 is the cutting force table for the

Table 2

Cutting force table obtained by cutting experiment

\begin{tabular}{llllrr}
\hline & & \multicolumn{5}{l}{ Feedrate $(\mathrm{mm} / \mathrm{min})$} \\
\cline { 2 - 6 } Cutting force $(\mathrm{N})$ & 50 & 100 & 150 & \multicolumn{1}{c}{200} \\
\hline$\delta_{\mathrm{e}}$ & $0.1(3 / 30)$ & 3.49 & 5.27 & 6.52 & 7.05 \\
& $0.2(6 / 30)$ & 4.52 & 6.73 & 8.36 & 9.56 \\
& $0.3(9 / 30)$ & 5.20 & 7.72 & 9.75 & 11.55 \\
& $0.4(12 / 30)$ & 5.62 & 8.59 & 10.47 & 12.53 \\
& $0.5(15 / 30)$ & 5.88 & 9.14 & 11.45 & 13.62 \\
& $1.0(30 / 30)$ & 6.61 & 10.14 & 12.94 & 16.35 \\
\hline
\end{tabular}

SCFM obtained by the experiment using a tool dynamometer (see Fig. 3), which will be used for the pocket machining given in Section 5. The cutter used in the experiment is a HSS (high speed steel) flat endmill having two flutes with a $60 \mathrm{~mm}$ diameter. The cutting material is pre-hardened steel, called HP4, of which the tensile strength is $90-100 \mathrm{~kg} / \mathrm{mm}^{2}$ and the hardness is 26-32 $\mathrm{H}_{\mathrm{R}} \mathrm{C}$. The axial depth-of-cut and RPM are $3 \mathrm{~mm}$ and 300, respectively. The level of the feedrate should be determined in the feasible range. In this experiment, a four-level feedrate is selected $(50,100,150$, $200 \mathrm{~mm} / \mathrm{min}$ ). On the other hand, the effective cutting depth is selected as the 2D chip-load, and a six-level cutting depth is selected $(0.1,0.2,0.3,0.4,0.5,1.0)$, where the maximum level of the cutting depth is set as the cutter radius of one based on our previous observation [10]: the cutting force does not increase if the cutting depth exceeds the cutter radius.

From the cutting force table obtained by the cutting experiment, the SCFM is constructed in the form of the non-parametric Bezier surface [11]. Shown in Fig. 4 is the SCFM generated from Table 2, where the $x$-, $y$-, and $z$-axis present the effective cutting depth, feedrate, and cutting force, respectively.

\section{Automatic feedrate adjustment procedure}

This section describes the AFA procedure that adjusts the feedrate of each NC block for maintaining the cutting force below a specified limit using a SCFM. As shown in Fig. 5, the procedure consists of the following three steps:

\subsection{Step 1. Generation of iso-cutting force curve}

This step generates the iso-cutting force curve of a cutting force limit from a SCFM. The cutting force limit is calculated from the nominal feedrate and nominal cutting depth, or the maximum allowable value for the cutter size, and the iso-cutting force curve is generated by the intersection operation between the non-parametric Bezier surface representing the SCFM and the horizontal plane having the $z$-axis value of the cutting force limit.

\subsection{Step 2. Calculation of peak-effective cutting depth}

This step calculates the peak-effective cutting depth of each concave line-line segment in the pocketing tool path 


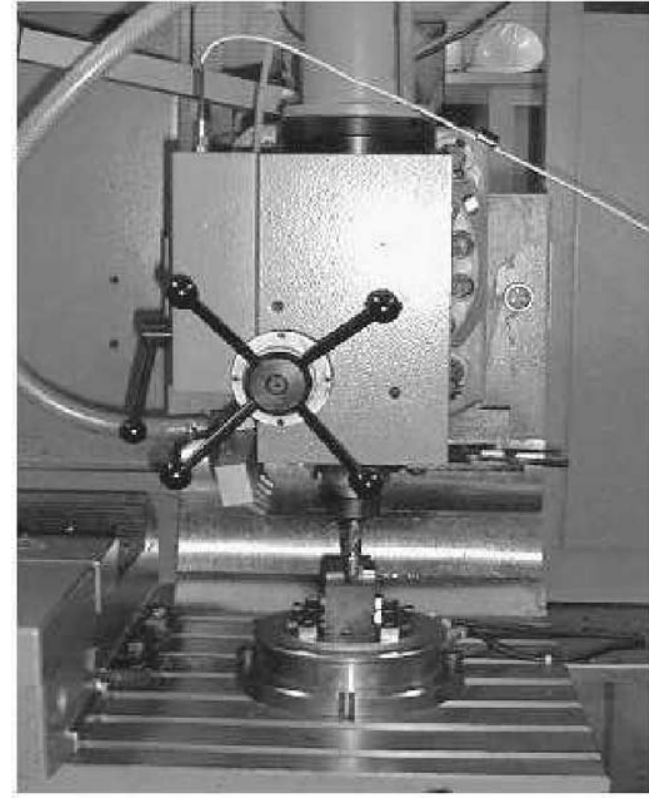

Fig. 3. Apparatus for cutting experiments.

via Eq. (1) and the peak cutter-engagement angle equations in Table 1.

\subsection{Step 3. Adjustment of feedrate}

This step determines the feedrate matching the peakeffective cutting depth in the iso-cutting force curve, and inserts an $\mathrm{NC}$ block corresponding to the transient range whose length is the braking distance. And to the other ranges of the original NC block, the nominal feedrate is assigned.

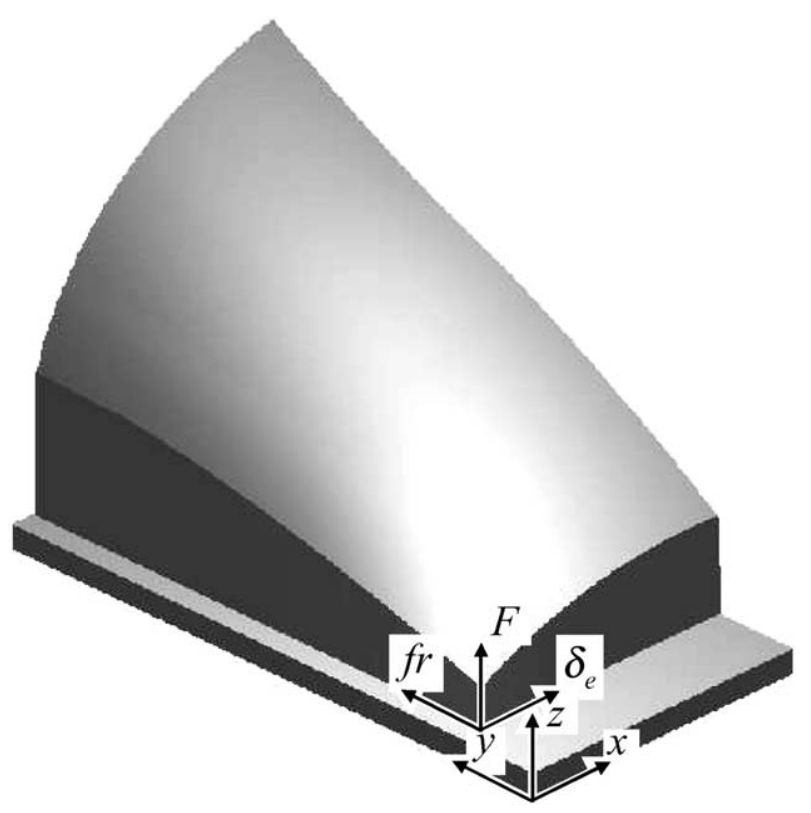

Fig. 4. Non-parametric Bezier surface representing SCFM.

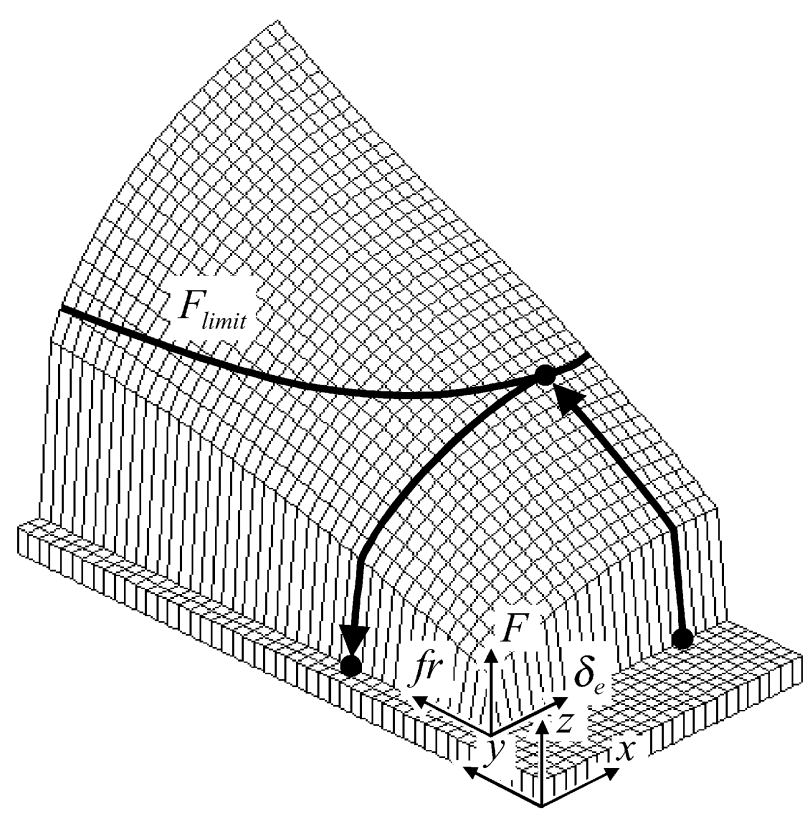

Fig. 5. Proposed AFA procedure.

\section{An illustrative example}

To show the applicability of the proposed method, this section gives an example of pocket machining. Shown in Fig. 6a is a CAD model, a TV front mask cabinet consisting of 247 trimmed surfaces where the width, length, and height of the CAD model are 840,730 , and $150 \mathrm{~mm}$, respectively. And shown in Fig. $6 \mathrm{~b}$ is the pocketing tool path generated on the cutting plane with a $z$-axis value of $15 \mathrm{~mm}$ below the top. To generate the tool path having the contourparallel offset (CPO) topology [12], the 2D cutting simulation approach [8] is used. The used tool is a flat endmill with a $60 \mathrm{~mm}$ diameter, and the path interval is $15 \mathrm{~mm}$ so that the nominal cutting depth is $15 \mathrm{~mm}$. The generated tool path consists of $2967 \mathrm{NC}$ blocks and its length is 22, $020.35 \mathrm{~mm}$.

In this case, an experienced $\mathrm{NC}$ operator set the feedrate at $155 \mathrm{~mm} / \mathrm{min}$ for the unmanned machining without any tool breakage or impaired surface quality, and the total machining time is $142.07 \mathrm{~min}$. Whereas, applying the proposed AFA procedure with the SCFM in the previous section, the total machining time is $118.89 \mathrm{~min}$. It is the saving of the machining time of $16.3 \%$ with respect to

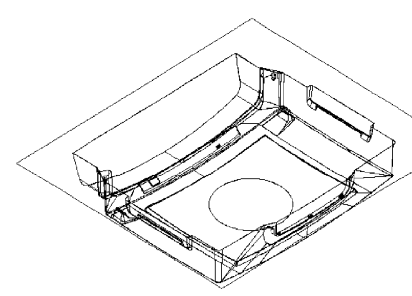

(a)

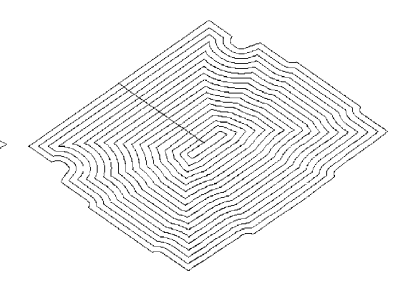

(b)
Fig. 6. An illustrative example of TV front mask cabinet. 


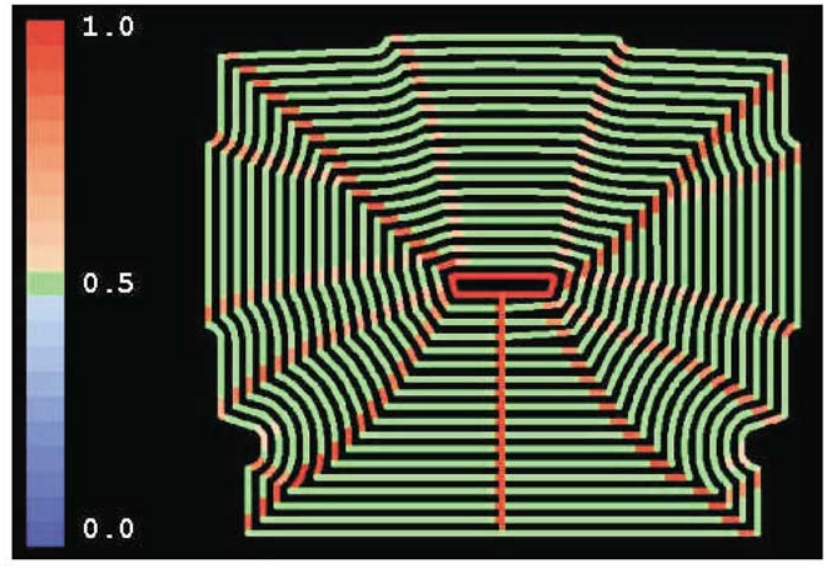

(a)

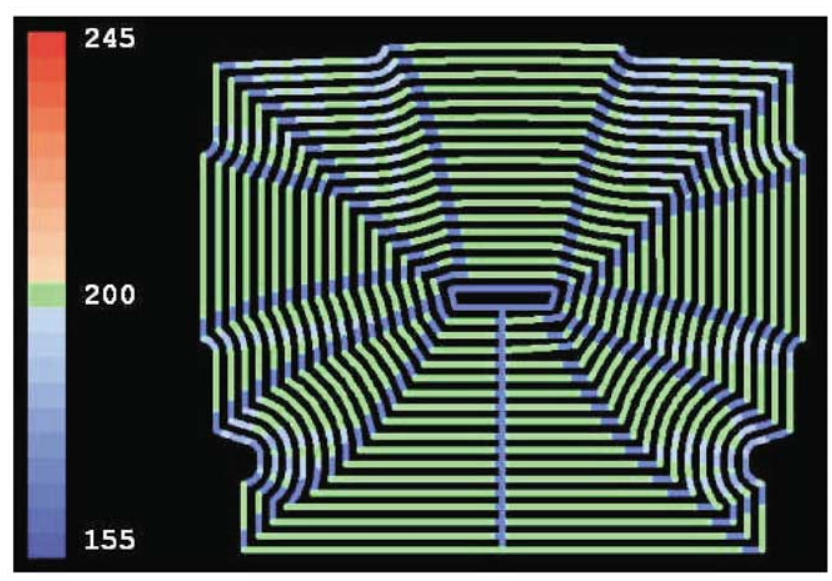

(b)

Fig. 7. Effective cutting depth and adjusted feedrate.

the machining without AFA. Clearly, the more the ratio of the total length of braking distances to that of the whole tool path decreases, the more the saving of the machining time (i.e. the effect of AFA) increases.

Shown in Fig. 7a is the 2D chip-load (i.e. the effective cutting depth) on the tool path. The effective cutting depth (normalized with respect to the cutter radius) ranges from 0.5 to 1 . Shown in Fig. $7 \mathrm{~b}$ is the adjusted feedrate by applying the proposed AFA procedure where the feedrate ranges $155-200 \mathrm{~mm} / \mathrm{min}$. The nominal values are indicated as green color in both the images. Fig. 7a and b look like the inverse images of each other because the lower feedrate, indicated by the blue color, is assigned to the region where the effective cutting depth is higher as indicated by the red color.

And shown in Fig. 8a is the image of resulting cutting force with the proposed AFA procedure. With the comparison to the cutting force image without AFA (see Fig. 8b), we can see that by applying the proposed AFA procedure, the cutting-load (or cutting force) is successfully regularized below the calculated cutting force limit $13.25 \mathrm{~N}$ (indicated by green color).

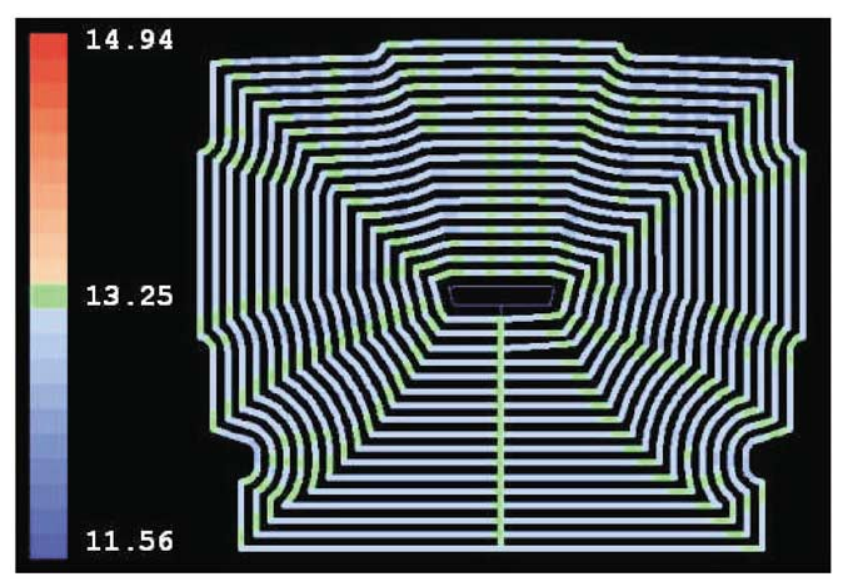

(a)

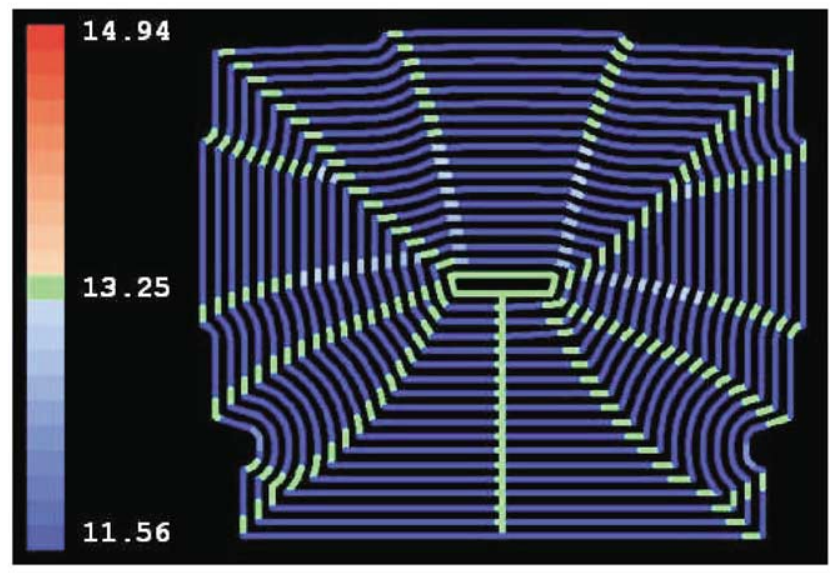

(b)

Fig. 8. Resulting cutting force images.

\section{Conclusion and discussion}

Proposed in the paper is an off-line approach type cuttingload regularization method for pocket machining. Like the conventional off-line approaches, the proposed method also requires a cutting force model, which is quite simplified with the function of two independent variables. One is the $2 \mathrm{D}$ chip-load (the cutter-engagement angle or effective cutting depth) and the other is the feedrate. The SCFM is easily obtained by the cutting experiment using a tool dynamometer for the given cutter and cutting material while some cutting parameters are specified by NC operators.

For pocket machining, we can assume the in-process shape of the part geometry is recognized without any cutting simulation. 2D chip-load analysis for the concave line-line segment shows the precise peak chip-load value and braking distance where the feedrate has to be reduced for the resulting cuttingload being smoothed. To assign the feedrate corresponding to the peak chip-load to the whole transient range looks somewhat 'conservative', however, the loss of the machining time can be ignored when the total length of braking distances is comparatively small to that of the whole tool path. 
The concept of the proposed method is simple, and the implementation is easy. Much time is not required to adjust the safe feedrate because there are no complex calculations as the infinitesimal flute model or no cutting simulation as the mean cutting force model. Nevertheless, the proposed method presents satisfactory results - the proposed method is more stable than the infinitesimal flute model and more precise than the mean cutting force model. The drawback of the proposed method is that the SCFM is not scalable and has to be reconstructed whenever the cutter and the cutting material are changed.

\section{Acknowledgements}

The Ministry of Science and Technology of Korea supported this research through a National Research Lab Project granted to Prof. Choi.

\section{References}

[1] Fussel BK, Srinivasan K. On-line identification of end milling process parameters. J Engng Industry 1989;111:322-30.

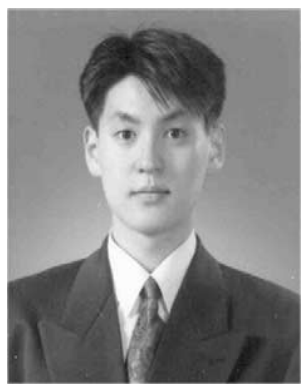

Seok-Hyung Bae is a $\mathrm{PhD}$ candidate in the Department of Industrial Engineering at Korea Advanced Institute of Science and Technology. He received a BS and MS in Mechanical Engineering from KAIST in 1994 and 1996, respectively. His research interests are in the area of surface modeling, CAD/CAM, and reverse engineering.

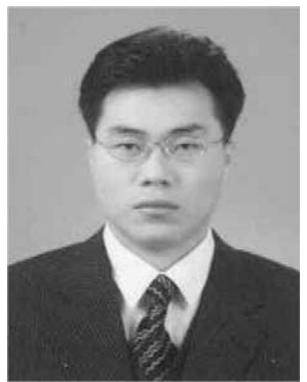

Bo H. Kim is a researcher at Korea Research Institute of Ships and Ocean Engineering. He received a BS from Chunnam National University, and an $\mathrm{MS}$ and $\mathrm{PhD}$ from Korea Advanced Institute of Science and Technology, all in Industrial Engineering. His research interests are in the area of sculptured surface machining, CAPP for die-cavity machining, development of intelligent CAM systems and virtual manufacturing.
[2] Asai A, Tsuruhashi T. Development of die-cutting feed rate control system. JSAE Rev 1988;9:72-82.

[3] Kline WA, DeVor DA, Lindberg JR. The prediction of cutting forces in end milling with application to cornering cuts. Int J Mach Tool Des Res 1982;22(1):7-22.

[4] Yang MY, Park HD. The prediction of cutting force in ball-end milling. Int J Mach Tool Manuf 1991;31(1):45-54.

[5] Automatic feedrate adjustment thins down machining time. American Machinist, April 1999. p. 38.

[6] Wang WP. Solid modeling for optimizing metal removal of threedimensional NC end milling. J Manuf Syst 1988;7(1):57-65.

[7] Tarng YS, Shyur YY, Lee BY. Computer-aided generation of the cutting conditions in pocket machining. J Mater Process Technol 1995;51:223-34.

[8] Choi BK, Kim BH. Die-cavity pocketing via cutting simulation. Comput-Aided Des 1997;29(12):837-46.

[9] Bae SH, Ko KH, Choi BK. Two-dimensional chip-load analysis for automatic feedrate adjustment. Trans Soc CAD/CAM Engnr 2000;5(2):155-67 in Korean.

[10] Ko KH, Seo JC, Choi BK. Automatic feedrate adjustment for profile milling. Trans Soc CAD/CAM Engnr 2000;5(2):175-83 in Korean.

[11] Choi BK. Surface modeling for CAD/CAM. Amsterdam: Elsevier, 1991 p. 334-6.

[12] Choi BK, Jerard R. Sculptured surface machining. Dordrecht: Kluwer Academic Publishers, 1998 p. 58-64.

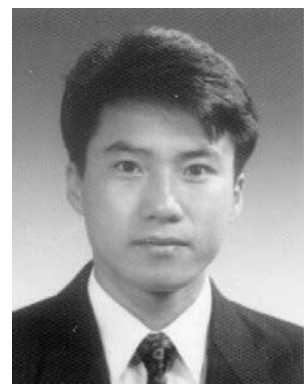

Keyhoon Ko is a PhD student in the department of industrial engineering at KAIST, where he got his MS degree as well. He received a BS from Sungkyunkwan University. His research interests are in the area of sculptured surface NC machining, CAPP for die-cavity machining, and development of intelligent CAM systems.

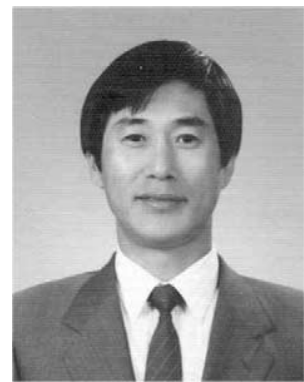

Byoung K. Choi is a professor of manufacturing systems engineering in the Department of Industrial Engineering at Korea Advanced Institute of Science and Technology since he joined KAIST in 1983. He received a BS from Seoul National University, a MS from KAIST, and a $\mathrm{PhD}$ from Purdue University, all in Industrial Engineering. His research interests are in the area of sculptured surface modeling, die-cavity machining, CAPP, system modeling and simulation, and virtual manufacturing. 